
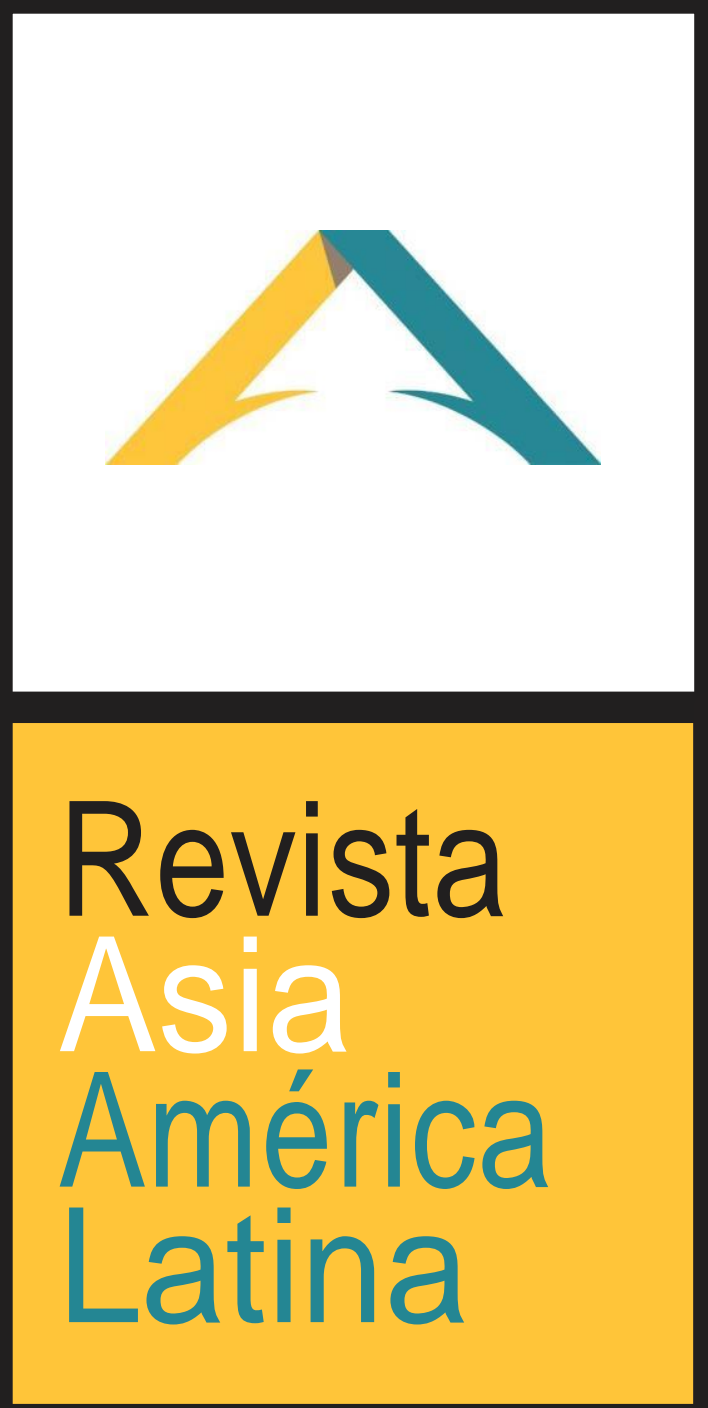

ISSN 2524-9347

Grupo de Estudios sobre Asia y América Latina Instituto de Estudios de América Latina y el Caribe Universidad de Buenos Aires

EU

DE BA 
MERCOSUR-ASEAN: las relaciones comerciales actuales y las oportunidades de negocio para el bloque latinoamericano en el sector de alimentos

SORAIA DANIELLE

\title{
MERCOSUR-ASEAN: LAS RELACIONES COMERCIALES ACTUALES Y LAS OPORTUNIDADES DE NEGOCIO PARA EL BLOQUE LATINOAMERICANO EN EL SECTOR DE ALIMENTOS
}

\author{
Soraia Danielle \\ Universidad Católica del Uruguay \\ Smar0804@gmail.com
}

\section{Introducción}

Ambos bloques tienen el componente del "regionalismo" como motor de inicio, a pesar de que hoy en día cada vez se aleje el pensamiento de firmar acuerdos regionales, sino que abiertos a cualquier parte del mundo. El Mercado Común del Sur (MERCOSUR) y la Asociación del de Naciones del Sudeste Asiático (ASEAN) proveyeron de un escenario de desarrollo e impacto positivo en sus respectivas regiones, provocaron un crecimiento del comercio intrarregional. Mientras que el bloque latinoamericano está enfrentando situaciones de conflicto que provocan que sus objetivos se atrasen y cambios de gobierno, ASEAN avanzó a grandes pasos en esta última década en materia económica-comercial impulsado por la firma de sus miembros en los megaacuerdos Comprehensive and Progressive Agreement for Trans-Pacific Partnership (CPATPP), Regional Comprehensive Economic Partnership (RCEP) y la cooperación Asia-Pacific Economic Cooperation (APEC).

La ASEAN fue fundada en 1967 con el ideal político y seguridad para proveer estabilidad y paz en la región, la cual siempre fue conflictiva por sus diferencias notorias, llegando a ser el escenario de uno de los conflictos más grandes de la Guerra Fría, la Guerra de Vietnam (1955-1975). Siendo así, los primeros países en firmar la Declaración de Bangkok (1984) que dio inicio al proceso de formación del bloque fueron Malasia, Tailandia, Singapur y Filipinas. Posteriormente Brunéi (1984), Vietnam (1995), Laos y Myanmar (1997) y la última adhesión fue Camboya (1999). Con ello conformaron una Asociación para la Cooperación Regional contemplando las heterogeneidades de sus miembros. Una vez logrado el objetivo de alcanzar la estabilidad, se prosiguió a una segunda etapa identificada como el impulso económicocomercial. En ella, se fundaron los cimientos para el surgimiento del nombre ASEAN en el escenario económico (Bartesaghi, 2014). Primero con la firma de la Zona de Libre Comercio en 1992, luego en el 2007 se reafirmó el pilar mencionado con la Carta de ASEAN (Bartesaghi \& Melgar, 2019). Siendo así, culmina con sus tres ejes principales que sostienen a la región: político y 
MERCOSUR-ASEAN: las relaciones comerciales actuales y las oportunidades de negocio para el bloque latinoamericano en el sector de alimentos

SORAIA DANIELLE

seguridad, económico y sociocultural (Asociación de Naciones del Sudeste Asiático, 2003).

Mientras que ASEAN formaba su Zona de Libre Comercio, en 1991 el MERCOSUR firma el Tratado de Asunción por sus miembros fundacionales: Argentina, Brasil, Paraguay y Uruguay con el ambicioso fin de formar a largo plazo un Mercado Común. El bloque fundamentalmente se formó por parte de Argentina y Brasil, quienes tenían una estrecha pero conflictiva relación comercial. Así como en la ASEAN también existía una relación de conflicto entre los miembros, a pesar de ser mucho más profunda. Llegando así a la gestación del bloque latinoamericano en conjunto con Uruguay y Paraguay, que aprovecharon para expandir sus negocios tanto intrarregionales como internacionales debido al aumento de competitividad en el escenario mundial que presentó su nueva imagen. Desde su inicio, el pilar de inserción internacional estuvo atado a sus principios de coordinación, al crear un arancel externo común y mencionándolo en el primer artículo del Tratado de Asunción como "la coordinación de posiciones en foros económico-comerciales-regionales e internacionales”. En términos de inserción internacional, volvió a retomar su pilar comercial con el nuevo Acuerdo con la Unión Europea pero no con el mismo impacto que hubiese tenido 20 años atrás, ya que existe una politización del bloque que también se reflejó en la firma de acuerdos; caso de Israel, Palestina, Egipto (María \& Pereira, 2016). Caso contrario al que sucede en el bloque del Sudeste Asiático, los Acuerdos que ha firmado sí representan los países con los cuales tienen destino sus principales exportaciones e importaciones. En el MERCOSUR solo durante la primera etapa firmó acuerdos de moderada importancia con países miembros de la ALADI.

Hoy en día la ASEAN es la sexta economía más potente del mundo ocupando el $7,3 \%$ del comercio mundial de importaciones en 2017. La economía crece a tasas del 5\% (Bartesaghi \& Melgar, 2019), convergiendo al total de una población estimada de 634 millones según el Banco Mundial. Lo que significa un amplio espectro de mercado para ingresar y grandes oportunidades de negocio a partir de las diferencias comerciales. Llegar a la ASEAN significará para el bloque su retomada de interés económico en el escenario mundial y volver a posicionarse en los estándares competitivos globales. Mirar hacia mercados externos es esencial para las regiones, contribuye a una mejora de la competitividad y un incremento del flujo de inversiones. Aun así, las diferencias entre ambos bloques en el entorno actual global del comercio son grandes, pero esto no se debe observar como un impedimento, sino como un factor a ser aprovechado y usado entre ambos para solucionar dicho obstáculo.

El objetivo del presente ensayo es analizar la situación actual en la que se encuentran ambos bloques comerciales de la región Sur-Sur, ASEAN y MERCOSUR, desarrollando la oportunidad de negocio que existe basándose 
MERCOSUR-ASEAN: las relaciones comerciales actuales y las oportunidades de negocio para el bloque latinoamericano en el sector de alimentos

en el fundamento de complementariedades. Por lo que se refiere a las oportunidades en exportación de alimentos, rubro por el cual el MERCOSUR posee ventaja competitiva (Perez, 2012). Abarcando los principales desafíos culturales que debe de superar para que las negociaciones vayan más allá de un TLC, sino que una cooperación a largo plazo.

\section{El escenario actual de las relaciones comerciales entre bloques}

En los últimos dos años las relaciones se profundizaron levemente entre ambos bloques y aumentaron los volúmenes de intercambio comercial con los miembros, principalmente con Brasil, el cual representa un gran interés para el Sudeste Asiático. Sin embargo, el primer movimiento significativo para el avance de un posible acuerdo fue durante la realización de la primera ronda de negociaciones MERCOSUR-Singapur. Por ser la primera reunión, acarreó los temas clásicos de un tratado comercial y, a su vez, algunos considerados avanzados: Bienes; Servicios e Inversiones; Reglas de Origen, Medidas Sanitarias y Fitosanitarias, Obstáculos Técnicos al Comercio, Competencia, Facilitación del Comercio, Compras Gubernamentales, Solución de Controversias, Propiedad Intelectual, y Defensa Comercial (Cancillería Argentina, 2019). Singapur es el principal bub logístico de la región, un importante factor que denota un esencial alcance a nuevos mercados inexplorados o con pocas exportaciones como el de Laos, del cual países como Uruguay y Paraguay no tienen participación. Singapur, ocupa el segundo puesto del ranking Doing Business 2018 de países con mayor facilidad de hacer negocios, tales como recepción de inversión extranjera, impuestos, trámites entre otros. Pero se ha dedicado a realizar inversiones significativas en otros países, lo cual es fundamental para que el bloque latinoamericano aumente su oferta de servicios, aumentar la generación de nuevos empleos e impulsar la mano de obra cualificada.

Brasil oficializó su interés por el bloque en el 2011, a partir de esa fecha las exportaciones aumentaron principalmente en el 2014 debido al precio de Residuos sólidos provenientes de la extracción de aceite de Soja (23.04.00) que según lo descripto en la nomenclatura, sirven para la preparación de animales. Como se puede observar en el gráfico, la balanza es favorable para el MERCOSUR. Pero se debe aclarar que el efecto en la balanza asimétrica para la ASEAN, no afecta en gran medida en su balanza comercial global. Ya que el MERCOSUR, como se puede observar en el siguiente gráfico, tiene un escaso impacto en sus importaciones y su foco principal son los intercambios comerciales con la Unión Europea y Estados Unidos. 
MERCOSUR-ASEAN: las relaciones comerciales actuales y las oportunidades de negocio para el bloque latinoamericano en el sector de alimentos

\section{Gráfico 1}

Comercio exterior entre el MERCOSUR y la ASEAN (Bienes)

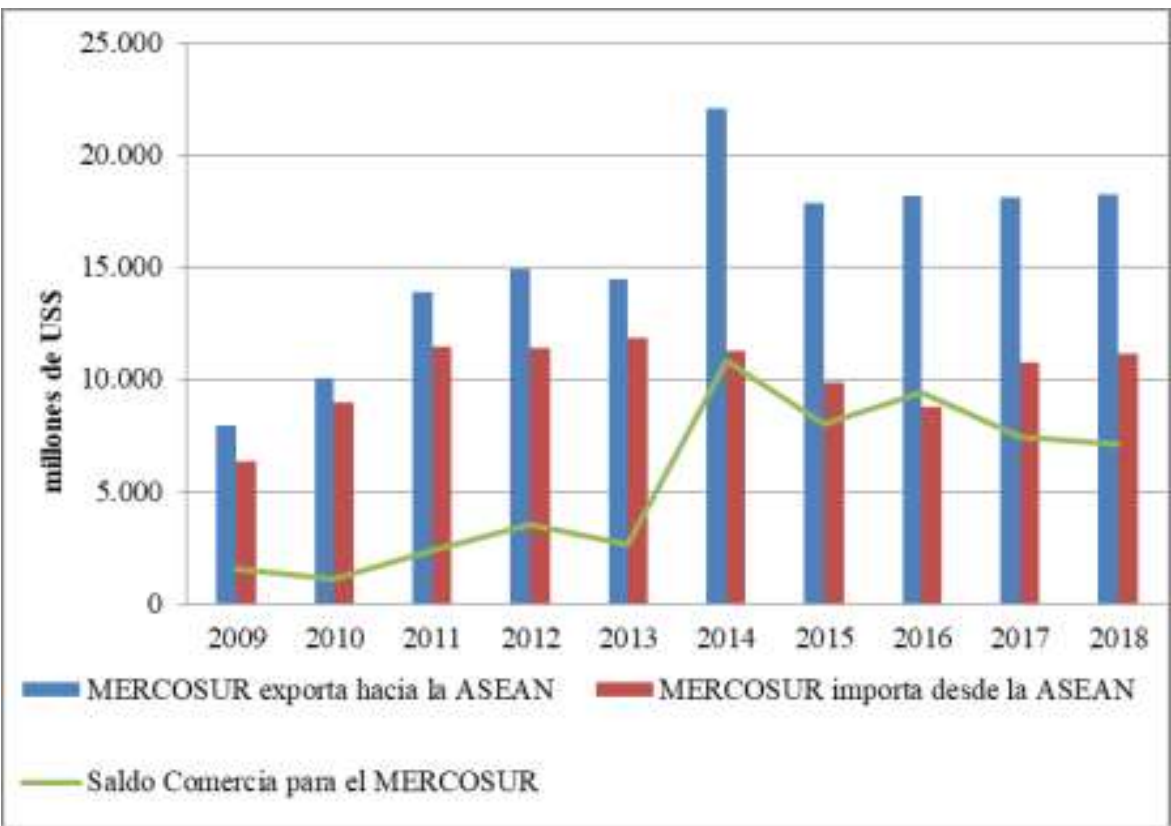

Fuente: elaboración propia en base a Trade Map

\section{Gráfico 2}

Participación de las exportaciones entre bloques

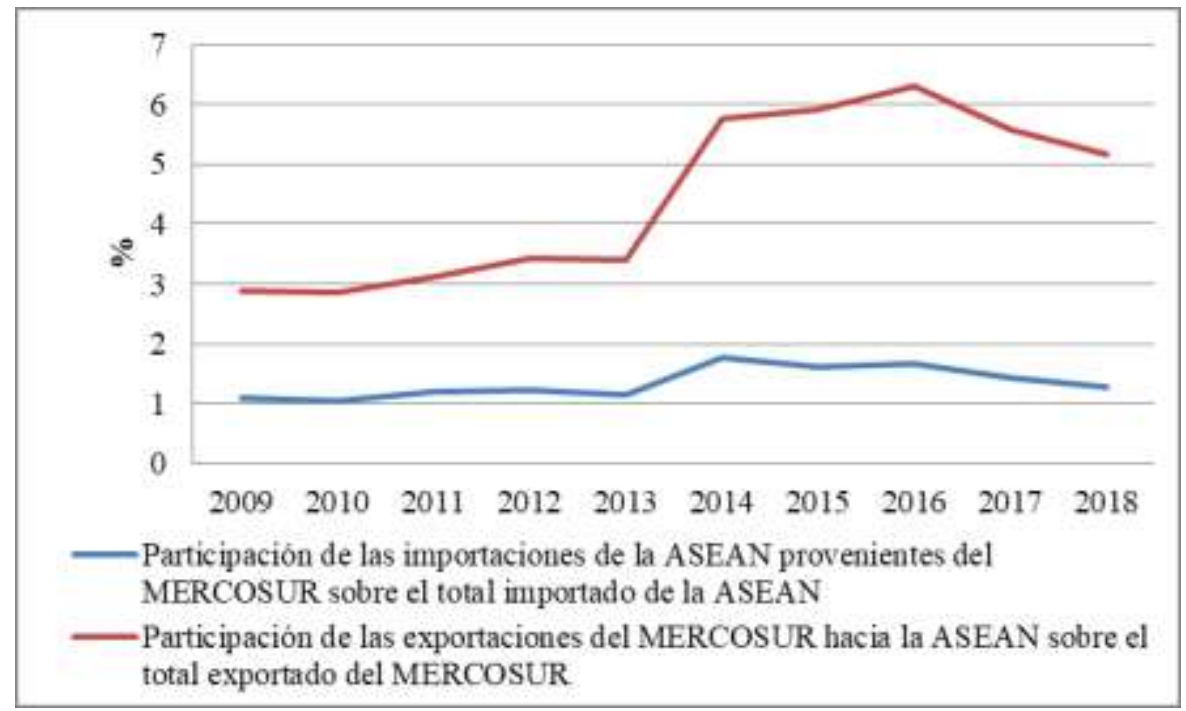

Fuente: elaboración propia en base a Trade Map 
MERCOSUR-ASEAN: las relaciones comerciales actuales y las oportunidades de negocio para el bloque latinoamericano en el sector de alimentos

El factor negativo de la balanza arraiga en la exportación de productos que son primarios y requieren poca mano de obra, en consecuencia, tienen poco valor agregado. A pesar de que en valores netos sean grandes volúmenes, no tiene gran presencia a nivel comercial, ya que son productos de fácil desvalorización en el mercado internacional. Englobadas las principales diez exportaciones del MERCOSUR como bloque, ocupan más del 80\% de las exportaciones totales, lo que significa que hay un concentrado muy grande en pocos productos. La tabla a continuación muestra la comparación de los productos exportados entre los bloques y se puede notar que los productos más exportados corresponden al sector de alimentos y derivados, con excepción del combustible y minerales. Ya en cuanto a las importaciones del MERCOSUR, se observa el agregado de valor que tienen los bienes, ubicándose en capítulos arancelarios avanzados.

\section{Tabla 1}

Comparación entre los productos exportados de la ASEAN y MERCOSUR en el marco de las relaciones entre bloque (Acumulado 2018)

\begin{tabular}{|c|c|c|c|c|c|c|}
\hline & \multicolumn{3}{|c|}{ ASEAN } & \multicolumn{3}{c|}{ MERCOSUR } \\
\hline $\begin{array}{c}\text { Ranki } \\
\text { ng }\end{array}$ & $\begin{array}{c}\text { Códi } \\
\text { go } \\
\text { SA }\end{array}$ & $\begin{array}{c}\text { Descripci } \\
\text { ón }\end{array}$ & $\begin{array}{c}\text { Participac } \\
\text { ión }\end{array}$ & $\begin{array}{c}\text { Códi } \\
\text { go } \\
\text { SA }\end{array}$ & $\begin{array}{c}\text { Descripció } \\
\text { n }\end{array}$ & $\begin{array}{c}\text { Participac } \\
\text { ión }\end{array}$ \\
\hline $\mathbf{1}$ & 85 & $\begin{array}{c}\text { Máquinas, } \\
\text { aparatos y } \\
\text { material } \\
\text { eléctrico }\end{array}$ & 32,38 & 23 & $\begin{array}{c}\text { Residuos y } \\
\text { desperdicios } \\
\text { de industrias } \\
\text { alimentarias }\end{array}$ & 28,12 \\
\hline $\mathbf{2}$ & 84 & $\begin{array}{c}\text { Máquinas, } \\
\text { aparatos y } \\
\text { artefactos } \\
\text { mecánicos }\end{array}$ & 15,75 & 10 & Cereales & 13,03 \\
\hline $\mathbf{3}$ & 87 & $\begin{array}{c}\text { Vehículos } \\
\text { automóvil } \\
\text { es, } \\
\text { tractores }\end{array}$ & 8,72 & 27 & $\begin{array}{c}\text { Combustible } \\
\text { minerales, } \\
\text { aceites } \\
\text { minerales }\end{array}$ & 12,37 \\
\hline $\mathbf{5}$ & 64 & $\begin{array}{c}\text { Cartuchos } \\
\text { y sus } \\
\text { manufact } \\
\text { uras }\end{array}$ & 7,79 & 26 & $\begin{array}{c}\text { Minerales } \\
\text { metalíferos, } \\
\text { escorias y } \\
\text { cenizas }\end{array}$ & 8,89 \\
\hline Calzado & 5,10 & 89 & Barcos & 6,82 \\
\hline
\end{tabular}


MERCOSUR-ASEAN: las relaciones comerciales actuales y las oportunidades de negocio para el bloque latinoamericano en el sector de alimentos

SORAIA DANIELLE

\begin{tabular}{|c|c|c|c|c|c|c|c|}
\hline $\begin{array}{l}\text { Asia } \\
\text { Annerica } \\
\text { Latina } \\
\end{array}$ & 6 & 15 & $\begin{array}{l}\text { Grasas y } \\
\text { sus } \\
\text { manufact } \\
\text { uras }\end{array}$ & 3,02 & 52 & Algodón & 3,89 \\
\hline 86 & 7 & 90 & $\begin{array}{c}\text { Instrumen } \\
\text { tos y } \\
\text { aparatos } \\
\text { de óptica }\end{array}$ & 2,91 & 02 & $\begin{array}{c}\text { Carne, } \\
\text { despojos } \\
\text { comestibles }\end{array}$ & 3,63 \\
\hline & 8 & 27 & $\begin{array}{l}\text { Combusti } \\
\text { bles } \\
\text { minerales, } \\
\text { aceites } \\
\text { minerales }\end{array}$ & 2,85 & 12 & $\begin{array}{l}\text { Semillas y } \\
\text { frutos } \\
\text { oleaginosos }\end{array}$ & 3,48 \\
\hline & 9 & 55 & $\begin{array}{c}\text { Fibras } \\
\text { sintéticas } \\
\text { o } \\
\text { Artificiale } \\
\text { s }\end{array}$ & 2,63 & 84 & $\begin{array}{l}\text { Máquinas } \\
\text { (turborreact } \\
\text { ores) }\end{array}$ & 2,75 \\
\hline & 10 & 39 & $\begin{array}{l}\text { Plásticos y } \\
\text { sus } \\
\text { manufact } \\
\text { uras }\end{array}$ & 2,22 & 41 & $\begin{array}{l}\text { Pieles y } \\
\text { cueros }\end{array}$ & 2,14 \\
\hline & \multicolumn{3}{|c|}{ Resto de los productos } & 16,61 & & & 14,89 \\
\hline
\end{tabular}

Fuente: elaboración propia en base a Trade Map.

La motivación para la ASEAN es Brasil por encontrarse entre los primero 30 exportadores. A continuación, se muestra una tabla en donde se puede observar la concentración existente en un único miembro del MERCOSUR, del total de las exportaciones, $67,7 \%$ son únicamente provenientes de Brasil.

Tabla 2

Participación de los Miembros del MERCOSUR en las Exportaciones hacia la ASEAN

\begin{tabular}{cccccc}
\hline País & Año & $\begin{array}{c}\text { Participación } \\
\mathbf{( \% )}\end{array}$ & Año & $\begin{array}{c}\text { Participación } \\
(\mathbf{\%})\end{array}$ & Variación \\
\hline Brasil & 2009 & 64,0 & 2017 & 67,7 & 3,7 \\
Argentina & 2009 & 33,8 & 2017 & 30,8 & $-3,0$ \\
Paraguay & 2009 & 1,0 & 2017 & 0,9 & $-0,1$
\end{tabular}


MERCOSUR-ASEAN: las relaciones comerciales actuales y las oportunidades de negocio para el bloque latinoamericano en el sector de alimentos

SORAIA DANIELLE

$\begin{array}{llllll}\text { Uruguay } & 2009 & 1,2 & 2017 & 0,6 & -0,5\end{array}$

Fuente: elaboración propia en base a Trade Map

Las inequidades existentes y la caída de las exportaciones de Argentina, significó un crecimiento en valor neto para Brasil. Por más que las exportaciones hayan tenido un aumento paulatino, siguen siendo los mismos productos. Solamente Uruguay en su vínculo bilateral con Indonesia, el 85\% está concentrado en dos productos madera y cueros (Bartesaghi, 2018). El último mencionado, está entre los productos más exportados al bloque asiático por parte del MERCOSUR.

Arraigado de la mano por las nuevas tendencias del negocio, un factor que debe considerarse como decisivo al momento de establecer una relación con el bloque ASEAN es la consideración de los nuevos temas OMC + y OMC X. Ya que el bloque del sudeste asiático es considerado avanzado y pionero en temas de negociación, porque no solo disputa sobre lo tradicional, que suelen ser puntos ya obligatorios en los acuerdos comerciales. Esto está arraigado en su mismo origen, los temas que siempre ha negociado son ene materia de cooperación, infraestructura, asuntos de carácter social, intercambio de conocimiento, entre otras. Como se mencionaba anteriormente, discuten temas que están por arriba de la Agenda del MERCOSUR, hoy en día Brasil está buscando socios que lo ayuden en materia de Transporte, Inversiones y Energías renovables, declarado por el secretario del Ministerio de Relaciones Exteriores Reinaldo José de Almeida.

En este asunto, se podría considerar a la Alianza del Pacífico como una fuerte amenaza al MERCOSUR, ya que sus miembros no solo realizan acuerdos comerciales mucho más rápido que el MERCOSUR. Sino que también realizan en base a los llamados de "nueva generación", en otras palabras, acuerdos de mayor alcance. Por ende, a pesar de que las exportaciones de Alianza Pacífico (AP) se encuentren en mejor posición, en cuanto a valor no alcanzan lo sumado entre Brasil y Argentina.

\section{Oportunidades de negocios}

Brasil es el exportador con mayor participación en el intercambio comercial con el bloque asiático. Es justo así, que en la presente sección donde se desarrollarán las oportunidades de negocio, se enfoque en los miembros: Argentina, Paraguay y Uruguay. Debido a la crisis en los países de principal exportación del MERCOSUR como China y la caída de los precios de los commodities, demostraron que la dependencia sobre escasos socios genera una inestabilidad, por eso se debe de persuadir en la diversificación de socios (Rubiolo, 2015). La diversificación de los socios comerciales es una 
MERCOSUR-ASEAN: las relaciones comerciales actuales y las oportunidades de negocio para el bloque latinoamericano en el sector de alimentos

oportunidad para ampliar el abanico de productos, así como el grado de autonomía y reducir los riesgos de limitarse en algunos países importadores. También explorar la elaboración de las Cadenas de Valor, ya que los países del bloque latinoamericano exportan en su mayoría materias primas con bajo valor de procesamiento.

Sin embargo, la primera posibilidad aparente de negocio que puede ser fructífera es la exportación de alimentos. Siendo una población de más de 650 millones, ASEAN representa una oportunidad para los exportadores de dicho rubro, como lo son los países mencionados, especialmente Uruguay que exporta fundamentalmente commodities. (Bartesaghi \& Melgar, 2019).

El enriquecimiento de la población fue dado por el aumento de la población joven, crecimiento económico de los ingresos medios, siendo que, en países como Malasia, Vietnam y Tailandia, la clase media es mayoría. Dicho hecho, cambia la configuración del consumo de bienes y servicios tanto en calidad como en cantidades. La demanda es creciente, representando una oportunidad de complementariedad que aún es inexplorada.

Cabe destacar, que a pesar de que los productos sean competitivos, ASEAN tiene una relación estrecha con Nueva Zelanda y Australia, con los cuales posee un TLC (Asean-Australia-New Zealand Free Trade Agreement o AANZFTA) que abarca la desgravación en alimentos. Ya que son exportadores de alta competencia en materia alimenticia, por consecuencia, accediendo sin aranceles o reducidos. El arancel promedio para Nación Más Favorecida, que abarca los productos primarios, tiene un promedio ponderado de 5,27\%. Los cereales, son el segundo producto más exportado por el MERCOSUR y el primero más exportado por Argentina a la ASEAN. Al momento de ingreso, tiene un arancel del 30\%. Mientras que Australia ingresa con un arancel del $0 \%$ debido a su tratado AANZFTA. En dicho rubro, existe una restricción en la oportunidad de negocio, pero se debe aprovechar que, en algunos de los productos, como los pertenecientes a los capítulos 23, 27, 59 los países del MERCOSUR ingresan con preferencia arancelaria del NMF, llegando a ser $0 \%$.

Residuos de soja y exportaciones de maíz, son los principales productos exportados por Argentina, debido a que el incremento del precio internacional de los commodities y el uso recurrente de nuevas tecnologías agrícolas proveyeron un mejor escenario a partir del 2011 (Rubiolo, 2017). Como se puede observar en el gráfico a continuación, la importación de alimento se ha más que quintuplicado desde el comienzo del siglo XXI, lo que significa que la población necesita abastecerse con insumos externos para poder cubrir los alimentos que no produce internamente. Este hecho está relacionado a motivos geográficos y climáticos que no permiten producir allí, por eso son clientes que cada vez comprarán más del resto del mundo. 
MERCOSUR-ASEAN: las relaciones comerciales actuales y las oportunidades de negocio para el bloque latinoamericano en el sector de alimentos

Gráfico 3

Importaciones de ASEAN para la categoría Alimentos (SA: 01 - 23)

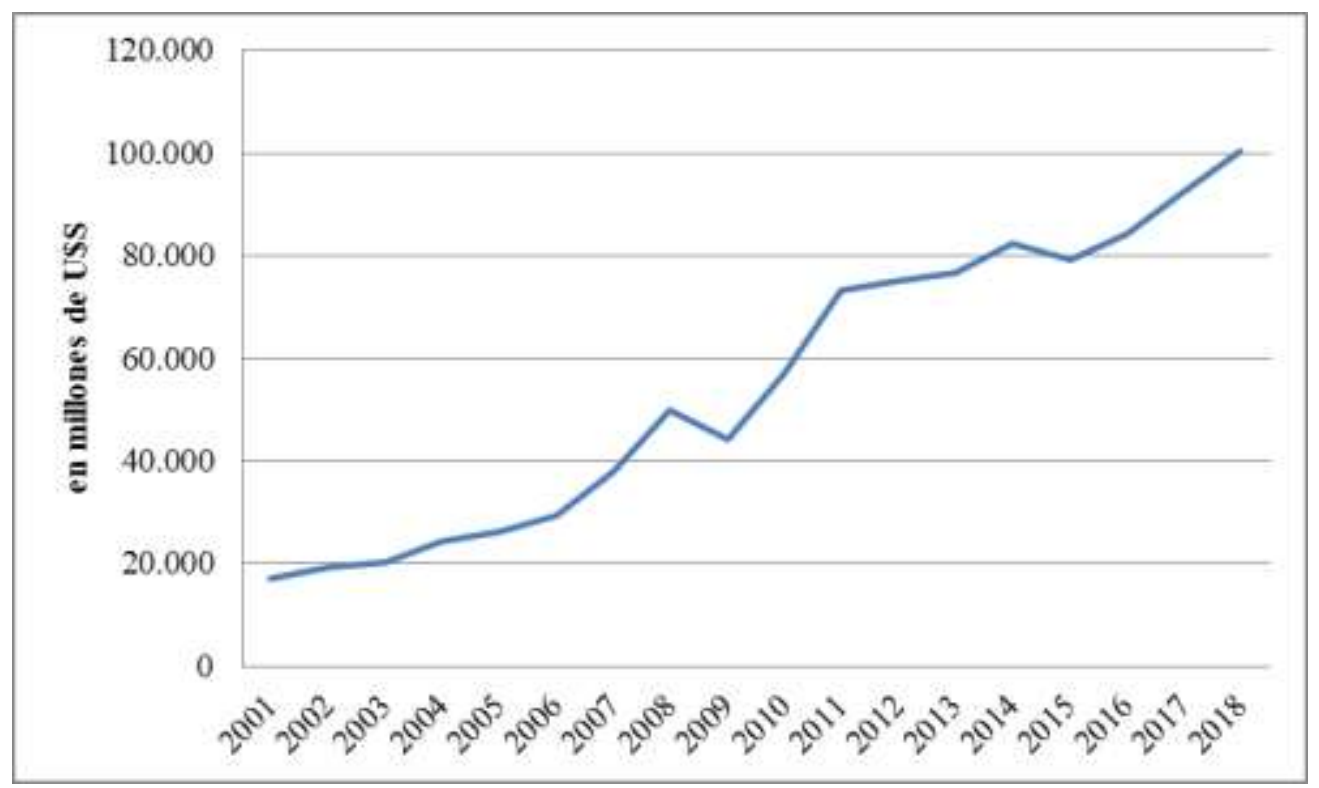

Fuente: elaboración propia en base a Trade Map

De comienzo las oportunidades deben estar enfocadas en los principales socios comerciales que tiene con el bloque del Sudeste Asiático, Singapur, Vietnam, Indonesia, Filipinas y Tailandia. Esto está manifestado de forma difusiva por parte de las recientes iniciativas que tuvo el MERCOSUR de acercarse a ellos, por un lado, las rondas de negociación para un futuro TLC con Singapur, las aperturas de embajadas de Uruguay y Paraguay en Indonesia. Llevando al caso, Indonesia se ha tornado cada vez más importante en la importación de productos que son competitivos en el MERCOSUR. Tales como la carne bovina, pescado, derivados de la leche como manteca, quesos y leche en polvo, este último que ha tenido aumento en el precio internacional. Además de los clásicos trigo, arroz, soja, azúcar, frutas y verduras. Al mismo tiempo, esto puede causar enfrentamientos en los países del bloque latinoamericano ya que intentarán todos colocar sus ofertas en los países, aprovechando las oportunidades (Bartesaghi, 2018). Para evitar estos enfrentamientos, es se podría establecer un sistema de cuotas, en donde cada país logre importar sus productos, sin perjudicar al otro.

Como se ha mencionado, hay una concentración en materia de producto, pero también en socios comerciales. Para ello se debe de diversificar la gama de socios comerciales, ya que por ejemplo en Uruguay casi el 60\% de las exportaciones se concentra en Vietnam y Tailandia (Bartesaghi \& Melgar, 
MERCOSUR-ASEAN: las relaciones comerciales actuales y las oportunidades de negocio para el bloque latinoamericano en el sector de alimentos

2019). Acotarse solo a la exportación de commodities refuerza el trabajo sobre la ventaja competitiva y no es un motor de innovación. Para ello se necesita de la cooperación entre bloques, para explorar nuevas oportunidades, que no se limiten a solamente un sector.

Más allá de las negociaciones en materias de bienes, se tiene conocimiento de que los países del Sudeste Asiático tienen una tendencia a la inversión, por lo que sería un efecto beneficioso para la balanza comercial desfavorable de la ASEAN. Reconocer el papel de la ciencia de la mano con la tecnología, es una oportunidad de intercambio de know-how y exportación mutua de servicios. Tanto Uruguay como Paraguay han desarrollado sus centros de software. Uruguay por su parte ha conseguido exportar servicios y atraer empresas internacionales para instalar outsourcing y offshoring.

\section{Desafíos culturales}

Para llegar al mercado asiático, se debe de sustentar una base sólida de intercambios culturales, información y cooperación. Las restricciones arancelarias y no arancelarias se superan luego de firmado un acuerdo, las culturales, no. Reducir la brecha cultural, que es uno de los tres pilares que sustenta el bloque del Sudeste Asiático, es uno de los principales desafíos que se encontrará a inicios de vinculación entre economías. Ya que el conocimiento mutuo y la confianza de una estabilidad son esenciales para las relaciones comerciales. A pesar de las distancias geográficas y culturales como el lingüístico, artístico, la aproximación reciente del MERCOSUR con algunos de los países miembros de la ASEAN ya acentuó un escenario favorable y pasible para las negociaciones. Aun así, falta mucho para estrechar un lazo firme, aprender los usos, costumbres y el estilo de negociación es el primer paso para llegar a un acercamiento tanto entre países como entre empresas.

Son mercados complejos de entrar y ágiles en negociación, actualmente existe una baja intensidad de acercamientos, por lo pronto no hay una meta en común de cooperación y un enlace que favorezca la formación de intereses comunes. A pesar de la ronda de negociación con Singapur, no se ha realizado un plan de acción por parte del MERCOSUR donde favorezca una estrategia o política de generación de conocimiento mutuo.

Solo en el marco de las exportaciones, hay un impacto directamente cultural que es la Certificación Halal. El 42\% de la población ASEAN es musulmana (Mufford, 2017), tener esta certificación es un requerimiento básico y de primera instancia para acceder al mercado. La compatibilización de los marcos de reglamentación surge pues, de ser una de las necesidades a concretar para el acceso en la materia de productos agrícolas y alimentos, que es en los capítulos que hay mayor compatibilidad entre los países, pero también mayores restricciones. 
MERCOSUR-ASEAN: las relaciones comerciales actuales y las oportunidades de negocio para el bloque latinoamericano en el sector de alimentos

Para realizar el acercamiento cultural, se puede entrelazar con las iniciativas de visitas comerciales, como lo hizo Argentina en el 2009 con el "Memorándum de entendimiento entra la República Argentina y el gobierno de Indonesia en materia de deportes". Acercó la asta del deporte para hacer conocer su país, en donde también incluyó el tango oriundo del Río de la Plata. También con acuerdos de cooperación, los cuales no necesariamente incluyen un nivel de profundidad alto y sirven para actuar en escenarios específicos como la Promoción de Inversión, intercambio de know-how. No sería completamente cierto, si se menciona que no hay acuerdos de cooperación, tratados y beneficios, ya que existen, pero no son explorados debido a la falta de interacción. En el ámbito del bloque solo hay uno firmado en el año de 2007 con Singapur que es el "Memorándum de entendimiento sobre Cooperación en materia de Comercio e Inversión y Plan de Acción"

Si se sigue por el ámbito bilateral, Brasil y Argentina, son los que tienen mayor cantidad de acuerdos de cooperación con los países de la ASEAN y en temáticas. Uruguay y Paraguay se encuentran por detrás, no tienen firmados en tantas materias. Pero en peor situación se encuentra Paraguay que tiene solo dos acuerdos, que son con Filipinas e Indonesia sobre la exención de visado, una temática que es básica. Brunéi, Camboya y Laos tienen una muy escasa interacción de acuerdos, solo con Argentina y Brasil que tienen algunos esporádicos durante los primeros años del siglo XXI. La cooperación más frecuente surge de la promoción y protección de inversiones recíprocas. Sólo Argentina tiene firmado bilateralmente con Filipinas, Indonesia, Tailandia y Vietnam. Ya Uruguay y Brasil tienen con Vietnam únicamente. Seguidamente surge la Cooperación Económica, Científica y Tecnológica, también en el ámbito bilateral que Uruguay se ha extendido en mayor medida con Malasia, Vietnam e Indonesia.

Uno de los que cabe destacar, es la cooperación oficial que hicieron entre dos centros de investigación de Argentina y Malasia: INTA (Instituto Nacional de Tecnología Agropecuaria) y MARDI (Malaysian Agricultural Research and Development Institute). En temas culturales, solo Argentina y Brasil tienen acuerdos con relación a la cooperación de entendimiento cultural, turístico y deportivo que se produjeron entre 2012 y 2019. Por ello, cuando se acerquen las rondas de negociaciones de ambos bloques, se encontrarán que cada país negociará los nuevos temas por su propia cuenta y no como una organización. Esto representa una seria desventaja para el contrincante asiático, que ya viene instaurando una armonización en toda la zona, y esto se debe a su pasado histórico de guerras, conflictos y su deseo por el desarrollo de las naciones en conjunto y no una disputa avasalladora. La presencia de empresas del MERCOSUR, así como las inversiones favorecerá el avance de las relaciones, también con incentivos como la reducción de costos ya sea de instalación como también aquellos decurrentes del comercio, como el flete. 
MERCOSUR-ASEAN: las relaciones comerciales actuales y las oportunidades de negocio para el bloque latinoamericano en el sector de alimentos

\section{Consideraciones finales}

Sobre el paso de los años, ambos bloques tuvieron un proceso invertido de desarrollo y profundización. Mientras que el bloque del Sudeste Asiático alcanzó su pilar de comercio exterior, el MERCOSUR se transformó en aquello que ASEAN usó en su provecho. El uso excesivo de la política en el bloque latinoamericano le significó grandes pérdidas de estabilidad en el escenario mundial sobre las cuestiones económicas y status. Llegar al ASEAN significará para el MERCOSUR no solo su retomada de interés del pilar económico en el contexto mundial, y seguir prosiguiendo con los principios que se establecieron desde su fundación y volver a posicionarse en los estándares competitivos. Mirar hacia mercados externos es esencial para las regiones, contribuye a una mejora de la competitividad, mejorando el precio de importación, incremento del flujo de inversiones, la diversificación de socios y el beneficio de diferentes actores sociales. Específicamente, la cooperación SurSur ha favorecido a países en desarrollo en materias de innovación y desarrollo en conjunto, con especial énfasis en América Latina y el Sudeste Asiático.

Para llegar al objetivo tan añorado, será necesario desarrollar un ambiente amigable de inversión en la región, así como también realizar promoción comercial, tales como las participaciones en ferias, visitas comerciales y misiones exploratorias, tornarlo atractivo, para así atraer inversores al territorio MERCOSUR. La apertura de Embajadas y Consulados también es una demostración de interés en la región, así como lo hicieron Uruguay y Paraguay.

Un hecho para tomar en cuenta, en favor al MERCOSUR, es que recientemente comenzó a realizar negociaciones con países que integran el foro ASEAN +3, lo cual representa cierta armonía entre un bloque y el otro. De igual forma, se declaró a finales de 2017 el interés de un posible TLC con Japón, las negociaciones no han sido retomadas, pero hay una declaración de inicio. Siguiendo por una misma línea de acción, durante el 2018 Uruguay, bilateralmente, hizo público un memorándum en el cual resaltaba el interés de comenzar las negociaciones para un TLC con China.

También se debe de tomar en cuenta que las próximas reuniones del MERCOSUR, en cuanto a su agenda externa, serán con Vietnam e Indonesia, los cuales son miembros de la ASEAN y acerca más aún un futuro encuentro entre bloques. Es una oportunidad, pero también un desafío, las negociaciones son diferentes por los estilos de cada país miembro pero también hay más beneficios a ser aprovechados. El próximo año de 2020, es un año crucial ya que culminará su ciclo establecido en el “ASEAN 2020” y abrirá uno nuevo con más proyectos de integración, posicionamiento de mercado y los ojos del mundo estarán allí. 
MERCOSUR-ASEAN: las relaciones comerciales actuales y las oportunidades de negocio para el bloque latinoamericano en el sector de alimentos

Este es un momento esencial para que el MERCOSUR diversifique sus socios comerciales en materia de comercio exterior, para disminuir el grado de dependencia que tiene de países como China que ocupan gran parte tanto de sus exportaciones como importaciones. No caer en la discontinuidad al estrechar lazos y fomentar un Plan de Acción a largo plazo tanto en el ámbito

multilateral como bilateral, para entrelazar los vínculos Sur-Sur fomentando los flujos de inversión y comercio.

\section{Referencias bibliográficas}

Association of Southeast Asian NAtions (2003). Declaration of ASEAN (Bali Concord II). https://asean.org/?static_post=declaration-ofasean-concord-ii-bali-concord-ii

BARTESAGHI, I. (2014). La ASEAN y el MERCOSUR. Similitudes, Diferencias y Potencialidades. Temas de Derecho Económico. Enfoque Nacional e

Internacional, $\mathrm{N}^{\circ} 2$.

https://ucu.edu.uy/sites/default/files/facultad/fce/dnii/La_ASEAN_ y_el_MERCOSUR_similit udes_diferencias_y_potencialidades.pdf

BARTESAGHI, I. (2018). Las relaciones comerciales entre Uruguay e Indonesia. Informe elaborado en el marco de la Cátedra Mercosur-ASEAN.

Bartesaghi, I., \& Melgar, N. (2019). Oportunidades de Uruguay en Asia: el caso de la Asociación de Naciones del Sudeste Asiático. Revista Diplomática 2 a Época. Vol. 1, pp. 83-92.

CAnCillería Argentina (26 de abril de 2019). Primera Ronda de Negociaciones MERCOSUR-Singapur.

https://www.cancilleria.gob.ar/es/actualidad/noticias/primera-rondade-negociaciones- mercosur-singapur

MARÍA, N. D., \& PEREIRA, M. E. (2016). Estrategias de inserción internacional del MERCOSUR y la ASEAN. Revista PUCE. Num. 103, pp. 273-297.

MufFord, T. L. (2017). A right for All: Freedom of religion or belief in Asian. United States Commission on International Religious Freedom. Washington, D.C.

PEREZ, M. (2012). Cooperación y Regionalismo entre MERCOSUR y la ASEAN Pacific. Buenos Aires: Universidad di Bologna - Representación en Buenos Aires.

Rubiolo, F. (2015). Argentina-Indonesia: Frotalecimiento de las relaciones comerciales y despertar del interés político en el marco de una estrategia Sur-Sur. Revista Ad-Minister, pp. 34-50.

Rubiolo, F. (2017). Argentina y el Sudeste de Asia en los noventa: análisis de una vinculación central para la actual estrategia Sur-Sur. Buenos Aires: Eduntref. 
Comité de ASEAN en Buenos Aires

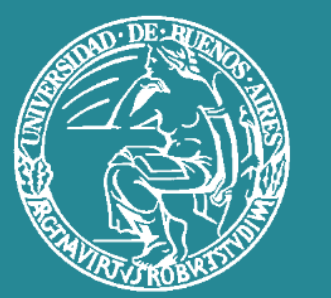

Grupo de Estudios sobre Asia y América Latina Instituto de Estudios de América Latina y el Caribe Universidad de Buenos Aires 